The Astrophysical Journal, 635:173-179, 2005 December 10

(C) 2005. The American Astronomical Society. All rights reserved. Printed in U.S.A.

\title{
IMAGING REDSHIFTS OF BL LACERTAE OBJECTS
}

\author{
B. Sbarufatti ${ }^{1}$ and A. Treves \\ Università dell'Insubria, Via Valleggio 11, I-22100 Como, Italy \\ AND \\ R. FALOMo \\ INAF, Osservatorio Astronomico di Padova, Vicolo dell'Osservatorio 5, I-35122 Padova, Italy \\ Received 2005 April 18; accepted 2005 August 2
}

\begin{abstract}
The HST snapshot imaging survey of 110 BL Lac objects (Urry et al.) has clearly shown that the host galaxies are massive and luminous ellipticals. The dispersion of the absolute magnitudes is sufficiently small that the measurement of the galaxy brightness becomes a valuable way of estimating their distance. This is illustrated by constructing a Hubble diagram of the 64 resolved objects with known redshift. By means of this relationship, we estimate the redshift of five resolved BL Lac objects of the survey that have no spectroscopic $z$. The adopted method also allows us to evaluate lower limits to the redshift for 13 objects of still unknown $z$ using the lower limit on the host galaxy magnitude. This technique can be applied to any BL Lac object for which an estimate or a lower limit of the host galaxy magnitude is available. Finally, we show that the distribution of the nuclear luminosity of all the BL Lac objects of the survey indicates that the objects for which both the redshift and the host galaxy are undetected are among the most luminous, and possibly the most highly beamed.
\end{abstract}

Subject heading: BL Lacertae objects: general

\section{INTRODUCTION}

Contrary to the large majority of active galactic nuclei (AGNs) characterized by optical spectra with prominent emission lines, BL Lac objects have quasi-featureless spectra. In fact, by the definition of this class of AGN, the line equivalent widths should be very small. The weakness of the spectral features has the consequence of hampering in many cases the determination of the redshift, thus making the distance of the source difficult to assess. As originally proposed by Blandford \& Rees (1978), the weakness of the spectral lines is most probably due to the fact that the underlying nonthermal continuum is exalted by the relativistic beaming of a jet pointing in the observer direction.

There are two possible strategies for deriving the distance (redshift) of BL Lac objects. The first is to improve the signal-tonoise ratio $(\mathrm{S} / \mathrm{N})$ of the optical spectra so that very weak spectral features may become detectable. At present, some significant progress with respect to the existing data can be obtained through the use of $8 \mathrm{~m}$ class telescopes (e.g., Heidt et al. 2004; Sbarufatti et al. 2005a, 2005b; Sowards-Emmerd et al. 2005).

The second approach requires high-quality imaging of the objects with the scope of detecting the host galaxy. Using it as a standard candle, one can obtain an albeit indirect estimate of the distance (e.g., Romanishin 1987; Falomo 1996; Falomo \& Kotilainen 1999; Heidt et al. 1999; Nilsson et al. 2003). For this approach one needs a combination of superb angular resolution and high efficiency in order to detect and characterize the faint extended light of the galaxy from the bright nucleus. These conditions become mandatory at high redshifts because of the dimming of the surface brightness, which scales as $(1+z)^{4}$.

The first imaging studies of BL Lac hosts were carried out using ground-based telescopes, which produced a preliminary characterization of the properties of host galaxies for various data sets (e.g., Abraham et al. 1991; Falomo 1996; Wurtz et al.

\footnotetext{
1 Also at Università di Milano-Bicocca.
}

1996; Falomo \& Kotilainen 1999; Heidt et al. 1999; Nilsson et al. 2003). These works have consistently shown that BL Lac hosts are virtually all massive elliptical galaxies (average luminosity $M_{R}=-23.7$ and effective radius $R_{e} \sim 10 \mathrm{kpc}$, assuming $H_{0}=$ $50 \mathrm{~km} \mathrm{~s}^{-1} \mathrm{Mpc}^{-1}, \Omega=0$ ). A substantial progress in this area was achieved through the use of Hubble Space Telescope (HST) images, and in particular by the BL Lac snapshot survey carried out with Wide Field Planetary Camera 2 (WFPC2). This produced high-quality homogeneous images for 110 objects (Falomo et al. 1997; Urry et al. 1999, 2000, hereafter U00; Scarpa et al. 2000a, 2000b; Falomo et al. 2000; O’Dowd \& Urry 2005).

In this paper we reconsider the results of the HST snapshot survey, with the aim of fully exploiting the information relevant for the determination of the distance of BL Lac objects. Our starting point is the analysis of the absolute magnitude distribution of the hosts. We show that since this distribution is relatively narrow, one can indeed use the host luminosity as a standard candle to evaluate the redshift. This is used in particular to determine the redshift of those objects for which the host galaxy is detected, but that still have featureless spectra. We then focus on the nuclei of the sources and show that the unresolved objects with pure featureless spectra are likely the most luminous and possibly beamed objects of the class.

\section{HST IMAGES OF BL LAC OBJECTS}

For this work we have considered the data set of images obtained by the HST BL Lac survey discussed by U00. It contains 110 objects imaged with HST WFPC2 in the F702W filter. The U00 sample was selected from seven flux-limited BL Lac samples in literature (1Jy, S4, PG, HEAO-A2, HEAO-A3, EMSS, and Slew). It includes both high-energy cutoff BL Lac (HBL) and low-energy cutoff BL Lac (LBL) objects (see Padovani \& Giommi 1995 for HBL and LBL definition), covering a wide range of jet physics in BL Lac objects. The surface brightness profile for each object was modeled with a combination of a 
TABLE 1

Host Galaxy and Nuclear Properties of BL Lac Objects

\begin{tabular}{|c|c|c|c|c|c|c|c|c|c|c|c|}
\hline $\begin{array}{l}\text { Object } \\
\text { (1) }\end{array}$ & $\begin{array}{l}\text { Type } \\
\text { (2) }\end{array}$ & $\begin{array}{l}\text { Group } \\
\text { (3) }\end{array}$ & $\begin{array}{l}A_{R} \\
\text { (4) }\end{array}$ & $\begin{array}{c}z \\
(5)\end{array}$ & $\begin{array}{c}\text { Galaxy } \\
K \text {-Correction } \\
\text { (6) }\end{array}$ & $\begin{array}{c}\text { Nucleus } \\
K \text {-Correction } \\
\text { (7) }\end{array}$ & $\begin{array}{l}\text { Evolution } \\
\text { Correction } \\
\quad(8)\end{array}$ & $\begin{array}{l}m_{n} \\
(9)\end{array}$ & $\begin{array}{c}M_{n} \\
(10)\end{array}$ & $\begin{array}{c}m_{h} \\
(11)\end{array}$ & $\begin{array}{c}M_{h} \\
(12)\end{array}$ \\
\hline $0033+595$ & $\mathrm{H}$ & $\mathrm{D}$ & 2.354 & {$[>0.24]$} & 0.71 & 0.06 & -0.39 & 18.23 & $<-24.25$ & $>20.00$ & $-22.90^{\mathrm{a}}$ \\
\hline $0118-272 \ldots \ldots \ldots \ldots \ldots \ldots \ldots$ & $\mathrm{L}$ & $\mathrm{C}$ & 0.036 & 0.559 & 0.91 & 0.14 & -0.43 & 15.78 & -26.96 & $>19.09$ & $>-23.86$ \\
\hline $0122+090$ & $\mathrm{H}$ & A & 0.249 & 0.339 & 0.43 & 0.10 & -0.28 & 21.98 & -19.65 & 18.88 & -22.67 \\
\hline 0138-097 …................. & $\mathrm{L}$ & $\mathrm{C}$ & 0.079 & 0.733 & 1.4 & 0.18 & -0.61 & 17.68 & -25.87 & $>20.19$ & $>-23.84$ \\
\hline $0158+001 \ldots \ldots \ldots \ldots \ldots \ldots$ & $\mathrm{H}$ & A & 0.064 & 0.298 & 0.36 & 0.08 & -0.24 & 18.38 & -22.72 & 18.27 & -22.75 \\
\hline $0229+200$ & $\mathrm{H}$ & A & 0.360 & 0.139 & 0.15 & 0.04 & -0.14 & 18.58 & -20.93 & 15.85 & -23.54 \\
\hline $0235+164$ & $\mathrm{~L}$ & $\mathrm{C}$ & 0.211 & 0.940 & 2.01 & 0.22 & -0.78 & 18.18 & -26.21 & $>19.75$ & $>-25.55$ \\
\hline $0257+342$ & $\mathrm{H}$ & A & 0.468 & 0.247 & 0.29 & 0.07 & -0.21 & 19.18 & -21.86 & 17.93 & -22.99 \\
\hline $0317+183 \ldots \ldots \ldots \ldots \ldots \ldots$ & $\mathrm{H}$ & A & 0.369 & 0.190 & 0.21 & 0.06 & -0.17 & 18.28 & -22.09 & 17.59 & -22.57 \\
\hline $0331-362 \ldots \ldots \ldots \ldots \ldots .$. & $\mathrm{H}$ & A & 0.050 & 0.308 & 0.38 & 0.09 & -0.25 & 19.03 & -22.15 & 17.81 & -23.28 \\
\hline 0347-121 ..................... & $\mathrm{H}$ & A & 0.125 & 0.188 & 0.21 & 0.06 & -0.17 & 18.28 & -21.74 & 17.72 & -22.17 \\
\hline 0350-371 ….............. & $\mathrm{H}$ & A & 0.020 & 0.165 & 0.18 & 0.05 & -0.16 & 18.03 & -21.60 & 17.08 & -22.38 \\
\hline 0414+009 ……............. & $\mathrm{H}$ & A & 0.314 & 0.287 & 0.35 & 0.08 & -0.24 & 16.08 & -25.19 & 17.49 & -23.67 \\
\hline 0419+194 …………....... & $\mathrm{H}$ & A & 1.476 & 0.512 & 0.79 & 0.13 & -0.40 & 19.53 & -24.44 & 21.05 & -23.02 \\
\hline $0426-380 \ldots \ldots \ldots \ldots \ldots . .$. & $\mathrm{L}$ & $\mathrm{C}$ & 0.066 & 1.105 & 2.44 & 0.24 & -0.94 & 18.08 & -26.61 & $>21.10$ & $>-24.80$ \\
\hline 0454+844 ……............... & $\mathrm{L}$ & $\mathrm{C}$ & 0.316 & 1.340 & 3.04 & 0.28 & -1.11 & 18.20 & -27.30 & $>22.37$ & $>-24.79$ \\
\hline $0502+675$ & $\mathrm{H}$ & A & 0.406 & 0.314 & 0.39 & 0.09 & -0.26 & 17.33 & -24.28 & 18.86 & -22.64 \\
\hline 0506-039 ................... & $\mathrm{H}$ & A & 0.220 & 0.304 & 0.37 & 0.09 & -0.25 & 18.73 & -22.61 & 18.35 & -22.87 \\
\hline $0521-365 \ldots \ldots \ldots \ldots \ldots \ldots$ & $\mathrm{L}$ & A & 0.105 & 0.055 & 0.06 & 0.02 & -0.06 & 15.28 & -21.99 & 14.60 & -22.43 \\
\hline 0537-441 „.................. & $\mathrm{L}$ & $\mathrm{C}$ & 0.101 & 0.896 & 1.89 & 0.21 & -0.74 & 15.83 & -28.30 & $>19.66$ & $>-25.32$ \\
\hline $0548-322$ ………........... & $\mathrm{H}$ & A & 0.094 & 0.069 & 0.07 & 0.02 & -0.08 & 16.93 & -20.68 & 14.62 & -22.89 \\
\hline $0607+710$ & $\mathrm{H}$ & A & 0.512 & 0.267 & 0.32 & 0.08 & -0.22 & 18.23 & -23.06 & 17.83 & -23.35 \\
\hline $0622-525 \ldots \ldots \ldots \ldots \ldots \ldots$ & $\mathrm{H}$ & $\mathrm{B}$ & 0.241 & {$[0.41]$} & 0.54 & 0.11 & -0.33 & 18.83 & -23.26 & 19.37 & $-22.90^{\mathrm{a}}$ \\
\hline $0647+250 \ldots \ldots \ldots \ldots \ldots \ldots$ & $\mathrm{H}$ & $\mathrm{D}$ & 0.264 & {$[>0.47]$} & 0.48 & 0.11 & -0.33 & 15.18 & $<-26.93$ & $>19.10$ & $-22.90^{\mathrm{a}}$ \\
\hline 0706+591 …................ & $\mathrm{H}$ & A & 0.103 & 0.125 & 0.14 & 0.04 & -0.13 & 17.53 & -21.54 & 15.94 & -22.95 \\
\hline $0715-259 \ldots \ldots \ldots \ldots \ldots .$. & $\mathrm{H}$ & A & 0.989 & 0.465 & 0.68 & 0.12 & -0.37 & 18.13 & -25.08 & 20.02 & -23.23 \\
\hline $0716+714$ & $\mathrm{H}$ & $\mathrm{D}$ & 0.082 & {$[>0.52]$} & 0.71 & 0.13 & -0.39 & 14.18 & $<-28.34$ & $>20.00$ & $-22.90^{\mathrm{a}}$ \\
\hline $0735+178$ & $\mathrm{~L}$ & $\mathrm{C}$ & 0.094 & 0.424 & 0.59 & 0.12 & -0.34 & 16.58 & -25.50 & $>20.44$ & $>-21.62$ \\
\hline $0737+744$ & $\mathrm{H}$ & A & 0.073 & 0.315 & 0.39 & 0.09 & -0.26 & 17.88 & -23.40 & 18.01 & -23.16 \\
\hline $0749+540$ & $\mathrm{~L}$ & $\mathrm{C}$ & 0.111 & 0.730 & 1.39 & 0.18 & -0.61 & 16.23 & -27.35 & $>21.78$ & $>-22.26$ \\
\hline 0754+100 .................... & $\mathrm{L}$ & $\mathrm{C}$ & 0.060 & 0.266 & 0.32 & 0.08 & -0.22 & 16.03 & -24.80 & $>18.69$ & $>-22.03$ \\
\hline $0806+524$ & $\mathrm{H}$ & A & 0.118 & 0.138 & 0.15 & 0.04 & -0.14 & 15.98 & -23.28 & 16.62 & -22.51 \\
\hline $0814+425 \ldots \ldots \ldots \ldots \ldots \ldots .$. & $\mathrm{L}$ & $\mathrm{D}$ & 0.170 & {$[>0.75]$} & 1.25 & 0.19 & -0.57 & 18.99 & $<-24.90$ & $>21.47$ & $-22.90^{\mathrm{a}}$ \\
\hline $0820+225 \ldots \ldots \ldots \ldots \ldots \ldots \ldots$ & $\mathrm{L}$ & $\mathrm{C}$ & 0.112 & 0.951 & 2.04 & 0.22 & -0.79 & 19.98 & -24.34 & $>21.90$ & $>-23.36$ \\
\hline $0823+033$ & $\mathrm{~L}$ & $\mathrm{C}$ & 0.122 & 0.506 & 0.78 & 0.13 & -0.40 & 17.78 & -24.79 & $>20.18$ & $>-22.49$ \\
\hline 0828+493 ……............... & $\mathrm{L}$ & A & 0.117 & 0.548 & 0.88 & 0.14 & -0.43 & 18.93 & -23.84 & 20.26 & -22.69 \\
\hline $0829+046 \ldots \ldots \ldots \ldots \ldots \ldots . .$. & $\mathrm{L}$ & A & 0.087 & 0.180 & 0.2 & 0.05 & -0.17 & 15.88 & -24.08 & 16.94 & -22.80 \\
\hline 0851+202 ……............ & $\mathrm{L}$ & $\mathrm{C}$ & 0.076 & 0.306 & 0.38 & 0.09 & -0.25 & 14.99 & -26.21 & $>18.53$ & $>-22.57$ \\
\hline $0922+749$ & $\mathrm{H}$ & A & 0.091 & 0.638 & 1.12 & 0.16 & -0.50 & 20.13 & -23.04 & 20.25 & -23.25 \\
\hline $0927+500$ & $\mathrm{H}$ & A & 0.045 & 0.188 & 0.21 & 0.06 & -0.17 & 17.48 & -22.46 & 17.62 & -22.19 \\
\hline 0954+658 ….................. & $\mathrm{L}$ & $\mathrm{C}$ & 0.306 & 0.367 & 0.48 & 0.10 & -0.30 & 16.08 & -25.81 & $>19.60$ & $>-22.24$ \\
\hline $0958+210 \ldots \ldots \ldots \ldots \ldots \ldots$ & $\mathrm{H}$ & A & 0.061 & 0.344 & 0.44 & 0.10 & -0.28 & 21.48 & -20.02 & 18.93 & -22.48 \\
\hline 1011+496 ……............... & $\mathrm{H}$ & A & 0.033 & 0.200 & 0.23 & 0.06 & -0.18 & 15.88 & -24.28 & 17.30 & -22.66 \\
\hline $1028+511$..................... & $\mathrm{H}$ & A & 0.033 & 0.361 & 0.47 & 0.10 & -0.29 & 16.48 & -25.13 & 18.55 & -22.97 \\
\hline $1044+549$ & $\mathrm{H}$ & $\mathrm{B}$ & 0.038 & {$[0.54]$} & 0.73 & 0.13 & -0.39 & 19.88 & -22.59 & 20.05 & $-22.90^{\mathrm{a}}$ \\
\hline $1104+384$ & $\mathrm{H}$ & A & 0.041 & 0.031 & 0.03 & 0.01 & -0.03 & 13.78 & -22.49 & 13.29 & -22.40 \\
\hline $1106+244$ & $\mathrm{H}$ & $\mathrm{B}$ & 0.045 & {$[0.46]$} & 0.59 & 0.13 & -0.33 & 18.28 & -24.20 & 19.57 & $-22.90^{\mathrm{a}}$ \\
\hline $1133+161$ …………........ & $\mathrm{H}$ & A & 0.173 & 0.460 & 0.67 & 0.12 & -0.37 & 20.28 & -22.11 & 19.83 & -22.57 \\
\hline $1136+704$ & $\mathrm{H}$ & A & 0.035 & 0.045 & 0.05 & 0.01 & -0.05 & 16.15 & -20.63 & 14.45 & -22.06 \\
\hline $1144-379 \ldots \ldots \ldots \ldots \ldots \ldots \ldots$ & $\mathrm{L}$ & $\mathrm{C}$ & 0.257 & 1.048 & 2.3 & 0.23 & -0.88 & 17.28 & -27.44 & $>23.03$ & $>-22.82$ \\
\hline $1147+245$ & $\mathrm{H}$ & $\mathrm{D}$ & 0.073 & {$[>0.63]$} & 0.95 & 0.15 & -0.46 & 16.87 & $<-26.13$ & $>20.70$ & $-22.90^{\mathrm{a}}$ \\
\hline 1207+394 „................... & $\mathrm{H}$ & A & 0.079 & 0.615 & 1.06 & 0.16 & -0.48 & 19.48 & -23.59 & 20.30 & -23.05 \\
\hline $1212+078$ & $\mathrm{H}$ & A & 0.059 & 0.136 & 0.15 & 0.04 & -0.14 & 16.38 & -22.82 & 16.02 & -23.02 \\
\hline $1215+303$ & $\mathrm{H}$ & A & 0.064 & 0.130 & 0.14 & 0.04 & -0.13 & 14.55 & -24.64 & 15.99 & -22.95 \\
\hline $1218+304$ & $\mathrm{H}$ & A & 0.056 & 0.182 & 0.2 & 0.05 & -0.17 & 15.68 & -24.25 & 17.12 & -22.62 \\
\hline $1221+245$ & $\mathrm{H}$ & A & 0.048 & 0.218 & 0.25 & 0.06 & -0.19 & 16.89 & -23.41 & 18.63 & -21.56 \\
\hline $1229+643$ & $\mathrm{H}$ & A & 0.049 & 0.164 & 0.18 & 0.05 & -0.16 & 18.03 & -21.63 & 16.38 & -23.09 \\
\hline $1239+069$ & $\mathrm{H}$ & $\mathrm{D}$ & 0.057 & {$[>0.92]$} & 1.63 & 0.21 & -0.75 & 18.45 & $<-25.66$ & $>22.30$ & $-22.90^{\mathrm{a}}$ \\
\hline $1246+586$ & $\mathrm{H}$ & $\mathrm{D}$ & 0.029 & {$[>0.73]$} & 1.14 & 0.17 & -0.57 & 15.66 & $<-27.72$ & $>21.20$ & $-22.90^{\mathrm{a}}$ \\
\hline $1248-296 \ldots \ldots \ldots \ldots \ldots \ldots$ & $\mathrm{H}$ & A & 0.202 & 0.370 & 0.49 & 0.10 & -0.30 & 18.83 & -23.02 & 18.87 & -22.89 \\
\hline $1249+174 \ldots \ldots \ldots \ldots \ldots \ldots$ & $\mathrm{H}$ & $\mathrm{C}$ & 0.058 & 0.644 & 1.14 & 0.16 & -0.51 & 18.51 & -24.66 & $>21.90$ & $>-21.60$ \\
\hline $1255+244$ & $\mathrm{H}$ & A & 0.034 & 0.141 & 0.15 & 0.04 & -0.14 & 17.08 & -22.25 & 16.72 & -22.38 \\
\hline
\end{tabular}


TABLE 1-Continued

\begin{tabular}{|c|c|c|c|c|c|c|c|c|c|c|c|}
\hline $\begin{array}{l}\text { Object } \\
\text { (1) }\end{array}$ & $\begin{array}{l}\text { Type } \\
\text { (2) }\end{array}$ & $\begin{array}{l}\text { Group } \\
\text { (3) }\end{array}$ & $\begin{array}{l}A_{R} \\
\text { (4) }\end{array}$ & $\begin{array}{c}z \\
(5)\end{array}$ & $\begin{array}{c}\text { Galaxy } \\
K \text {-Correction } \\
\text { (6) }\end{array}$ & $\begin{array}{c}\text { Nucleus } \\
K \text {-Correction } \\
\text { (7) }\end{array}$ & $\begin{array}{c}\text { Evolution } \\
\text { Correction } \\
\text { (8) }\end{array}$ & $\begin{array}{l}m_{n} \\
(9)\end{array}$ & $\begin{array}{c}M_{n} \\
(10)\end{array}$ & $\begin{array}{c}m_{h} \\
(11)\end{array}$ & $\begin{array}{c}M_{h} \\
(12)\end{array}$ \\
\hline $1402+041 \ldots \ldots \ldots \ldots$ & $\mathrm{H}$ & $\mathrm{C}$ & 0.069 & 0.340 & 0.43 & 0.10 & -0.28 & 16.38 & -25.12 & $>19.38$ & $>-22.00$ \\
\hline $1407+595$ & $\mathrm{H}$ & A & 0.037 & 0.495 & 0.75 & 0.13 & -0.39 & 18.84 & -23.59 & 19.04 & -23.47 \\
\hline $1418+546 \ldots \ldots \ldots \ldots \ldots \ldots$ & $\mathrm{L}$ & A & 0.036 & 0.152 & 0.17 & 0.05 & -0.15 & 15.68 & -23.82 & 16.10 & -23.18 \\
\hline $1422+580 \ldots \ldots \ldots \ldots \ldots \ldots$ & $\mathrm{H}$ & $\mathrm{C}$ & 0.027 & 0.683 & 1.25 & 0.17 & -0.55 & 18.35 & -24.95 & $>21.99$ & $>-21.71$ \\
\hline $1424+240 \ldots \ldots \ldots \ldots \ldots \ldots$ & $\mathrm{H}$ & $\mathrm{D}$ & 0.156 & {$[>0.67]$} & 1.06 & 0.17 & -0.57 & 14.66 & $<-28.85$ & $>21.00$ & $-22.90^{\mathrm{a}}$ \\
\hline $1426+428 \ldots \ldots \ldots \ldots \ldots$ & $\mathrm{H}$ & A & 0.033 & 0.129 & 0.14 & 0.04 & -0.13 & 17.38 & -21.63 & 16.14 & -22.75 \\
\hline $1437+398 \ldots \ldots \ldots \ldots \ldots$ & $\mathrm{L}$ & B & 0.037 & {$[0.26]$} & 0.29 & 0.09 & -0.18 & 16.73 & -24.43 & 17.95 & $-22.90^{\mathrm{a}}$ \\
\hline $1440+122 \ldots \ldots \ldots \ldots \ldots$ & $\mathrm{H}$ & A & 0.076 & 0.162 & 0.18 & 0.05 & -0.15 & 16.93 & -22.75 & 16.71 & -22.77 \\
\hline $1458+224 \ldots \ldots \ldots \ldots \ldots$ & $\mathrm{H}$ & A & 0.128 & 0.235 & 0.27 & 0.07 & -0.20 & 15.78 & -24.82 & 17.80 & -22.65 \\
\hline $1514-241$................... & $\mathrm{H}$ & A & 0.369 & 0.049 & 0.05 & 0.02 & -0.05 & 14.48 & -22.65 & 14.45 & -22.58 \\
\hline $1517+656 \ldots \ldots \ldots \ldots \ldots$ & $\mathrm{H}$ & $\mathrm{C}$ & 0.068 & 0.702 & 1.3 & 0.17 & -0.58 & 16.18 & -27.24 & $>19.89$ & $>-23.94$ \\
\hline $1519-273 \ldots \ldots \ldots \ldots \ldots$ & $\mathrm{L}$ & $\mathrm{C}$ & 0.636 & 1.297 & 2.92 & 0.27 & -1.09 & 17.03 & -28.69 & $>20.40$ & $>-26.88$ \\
\hline $1533+535 \ldots \ldots \ldots \ldots \ldots$ & $\mathrm{H}$ & $\mathrm{C}$ & 0.051 & 0.890 & 1.87 & 0.21 & -0.74 & 17.88 & -26.19 & $>19.70$ & $>-25.19$ \\
\hline $1534+014$ & $\mathrm{H}$ & A & 0.152 & 0.312 & 0.39 & 0.09 & -0.25 & 19.08 & -22.27 & 18.16 & -23.08 \\
\hline $1538+149 \ldots \ldots \ldots \ldots \ldots$ & $\mathrm{L}$ & A & 0.148 & 0.605 & 1.03 & 0.15 & -0.46 & 17.94 & -25.14 & 20.22 & -23.14 \\
\hline $1544+820 \ldots \ldots \ldots \ldots \ldots . .$. & $\mathrm{H}$ & $\mathrm{D}$ & 0.133 & {$[>0.46]$} & 0.60 & 0.13 & -0.33 & 16.55 & $<-26.02$ & $>19.60$ & $-22.90^{\mathrm{a}}$ \\
\hline $1553+113 \ldots \ldots \ldots \ldots \ldots \ldots \ldots$ & $\mathrm{H}$ & $\mathrm{D}$ & 0.139 & {$[>0.78]$} & 1.31 & 0.21 & -0.68 & 14.43 & $<-29.76$ & $>21.60$ & $-22.90^{\mathrm{a}}$ \\
\hline $1704+604 \ldots \ldots \ldots \ldots \ldots$ & $\mathrm{H}$ & A & 0.062 & 0.280 & 0.33 & 0.08 & -0.23 & 21.08 & -19.92 & 18.69 & -22.15 \\
\hline $1722+119 \ldots \ldots \ldots \ldots \ldots \ldots$ & $\mathrm{H}$ & $\mathrm{D}$ & 0.458 & {$[>0.68]$} & 1.23 & 0.17 & -0.57 & 14.61 & $<-29.20$ & $>21.40$ & $-22.90^{\mathrm{a}}$ \\
\hline $1728+502 \ldots \ldots \ldots \ldots \ldots$ & $\mathrm{H}$ & A & 0.079 & 0.055 & 0.06 & 0.02 & -0.06 & 16.43 & -20.81 & 15.49 & -21.51 \\
\hline $1738+476 \ldots \ldots \ldots \ldots \ldots$ & $\mathrm{L}$ & $\mathrm{D}$ & 0.050 & {$[>0.60]$} & 0.87 & 0.15 & -0.39 & 19.63 & $<-23.35$ & $>20.50$ & $-22.90^{\mathrm{a}}$ \\
\hline $1745+504 \ldots \ldots \ldots \ldots \ldots$ & $\mathrm{H}$ & B & 0.086 & {$[0.46]$} & 0.59 & 0.13 & -0.33 & 21.18 & -21.34 & 19.57 & $-22.90^{\mathrm{a}}$ \\
\hline $1749+096 \ldots \ldots \ldots \ldots$ & $\mathrm{L}$ & A & 0.482 & 0.320 & 0.4 & 0.09 & -0.26 & 16.88 & -24.88 & 18.82 & -22.81 \\
\hline $1749+701 \ldots \ldots \ldots \ldots \ldots$ & $\mathrm{L}$ & $\mathrm{C}$ & 0.083 & 0.770 & 1.51 & 0.19 & -0.65 & 15.83 & -27.87 & $>19.28$ & $>-24.96$ \\
\hline $1757+703 \ldots \ldots \ldots \ldots \ldots$ & $\mathrm{H}$ & A & 0.089 & 0.407 & 0.56 & 0.11 & -0.33 & 18.43 & -23.52 & 19.58 & -22.35 \\
\hline $1803+784 \ldots \ldots \ldots \ldots \ldots$ & $\mathrm{L}$ & $\mathrm{C}$ & 0.140 & 0.684 & 1.25 & 0.17 & -0.56 & 16.18 & -27.24 & $>20.89$ & $>-22.91$ \\
\hline $1807+698 \ldots \ldots \ldots \ldots \ldots \ldots$ & $\mathrm{L}$ & A & 0.096 & 0.051 & 0.05 & 0.02 & -0.06 & 14.95 & -22.30 & 13.87 & -22.97 \\
\hline $1823+568 \ldots \ldots \ldots \ldots \ldots \ldots$ & $\mathrm{L}$ & A & 0.164 & 0.664 & 1.2 & 0.17 & -0.53 & 18.29 & -25.07 & 20.24 & -23.49 \\
\hline $1853+671 \ldots \ldots \ldots \ldots \ldots$ & $\mathrm{H}$ & A & 0.121 & 0.212 & 0.24 & 0.06 & -0.19 & 19.48 & -20.88 & 18.19 & -21.99 \\
\hline 1914-194 …................ & $\mathrm{H}$ & A & 0.345 & 0.137 & 0.15 & 0.04 & -0.14 & 15.30 & -24.19 & 16.95 & -22.39 \\
\hline $1959+650 \ldots \ldots \ldots \ldots \ldots$ & $\mathrm{H}$ & A & 0.473 & 0.048 & 0.05 & 0.02 & -0.05 & 15.38 & -21.85 & 14.92 & -22.17 \\
\hline $2005-489$..................... & $\mathrm{H}$ & A & 0.149 & 0.071 & 0.07 & 0.02 & -0.08 & 12.73 & -25.23 & 14.52 & -23.11 \\
\hline $2007+777 \ldots \ldots \ldots \ldots \ldots$ & $\mathrm{L}$ & A & 0.431 & 0.342 & 0.44 & 0.10 & -0.28 & 18.03 & -23.84 & 19.03 & -22.73 \\
\hline $2037+521 \ldots \ldots \ldots \ldots \ldots$ & $\mathrm{H}$ & A & 2.445 & 0.053 & 0.05 & 0.02 & -0.06 & 19.48 & -20.12 & 16.15 & -23.12 \\
\hline $2131-021 \ldots \ldots \ldots \ldots \ldots$ & $\mathrm{L}$ & $\mathrm{C}$ & 0.147 & 1.285 & 2.89 & 0.27 & -1.08 & 19.00 & -26.20 & $>21.98$ & $>-24.76$ \\
\hline $2143+070 \ldots \ldots \ldots \ldots \ldots$ & $\mathrm{H}$ & A & 0.200 & 0.237 & 0.27 & 0.07 & -0.20 & 18.21 & -22.46 & 17.89 & -22.66 \\
\hline $2149+173 \ldots \ldots \ldots \ldots \ldots \ldots$ & $\mathrm{L}$ & $\mathrm{D}$ & 0.270 & {$[>0.76]$} & 1.31 & 0.19 & -0.68 & 18.63 & $<-25.36$ & $>21.60$ & $-22.90^{\mathrm{a}}$ \\
\hline $2200+420 \ldots \ldots \ldots \ldots \ldots$ & $\mathrm{L}$ & A & 0.880 & 0.069 & 0.07 & 0.02 & -0.08 & 13.58 & -24.82 & 15.37 & -22.93 \\
\hline $2201+044 \ldots \ldots \ldots \ldots \ldots \ldots$ & $\mathrm{L}$ & A & 0.113 & 0.027 & 0.03 & 0.01 & -0.03 & 17.18 & -18.54 & 13.74 & -21.72 \\
\hline $2240-260 \ldots \ldots \ldots \ldots \ldots$ & $\mathrm{L}$ & $\mathrm{C}$ & 0.057 & 0.774 & 1.53 & 0.19 & -0.65 & 17.53 & -26.15 & $>22.08$ & $>-22.17$ \\
\hline $2254+074 \ldots \ldots \ldots \ldots \ldots$ & $\mathrm{L}$ & A & 0.176 & 0.190 & 0.21 & 0.06 & -0.17 & 16.94 & -23.24 & 16.61 & -23.36 \\
\hline $2326+174 \ldots \ldots \ldots \ldots \ldots$ & $\mathrm{H}$ & A & 0.150 & 0.213 & 0.24 & 0.06 & -0.19 & 17.63 & -22.76 & 17.56 & -22.66 \\
\hline $2344+514 \ldots \ldots \ldots \ldots \ldots \ldots$ & $\mathrm{H}$ & A & 0.577 & 0.044 & 0.04 & 0.01 & -0.05 & 16.83 & -20.49 & 14.01 & -22.98 \\
\hline $2356-309$ & $\mathrm{H}$ & A & 0.036 & 0.165 & 0.18 & 0.05 & -0.16 & 17.28 & -22.37 & 17.21 & -22.27 \\
\hline
\end{tabular}

Notes.-Col. (1): Object name. Col. (2): Object type. H: HBL, L: LBL. Col. (3): Object group. A: Host galaxy detected, redshift known; B: host detected, redshift unknown; C: host not detected, redshift known; D: host not detected, redshift unknown. Col. (4): Galactic extinction coefficient by Schlegel et al. (1998). Col. (5): Redshift. Square brackets indicate imaging redshifts and redshift lower limits from imaging. Col. (6): Host galaxy $K$-correction by Poggianti (1997). Col. (7): Nucleus $K$-correction, calculated assuming $F_{\lambda} \propto \lambda^{-0.7}$. Col. (8): Host galaxy evolution correction. Col. (9): Nucleus apparent $R$ magnitude. Col. (10): Nucleus absolute $R$ magnitude. Col. (11): Host galaxy apparent $R$ magnitude. Col. (12): Host galaxy absolute $R$ magnitude.

${ }^{\text {a }}$ Host galaxy absolute magnitude is assumed to be $M_{R}=M_{R}^{\text {fit }}=-22.9$ in order to obtain a redshift estimate.

point source (the nucleus component), and an extended emission (the host galaxy) represented by an elliptical (de Vaucouleurs law) or a disk component (exponential law). For all the sources whose host galaxies can be detected, the radial brightness profile is consistent with the host being an elliptical. For the unresolved sources, U00 give a lower limit on the apparent magnitude of the underlying nebulosity.

In order to construct a more homogeneous and updated data set of BL Lac host galaxies measurements, we have introduced a number of revisions in the treatment of the results of U00, which are listed in the following:
1. For three objects $0145+138,0446+449$, and $0525+713$, there is no evidence of a nuclear component in the HST images. This makes the classification of BL Lac dubious, and therefore they are not considered here. The object $1320+$ 084 has been excluded from the sample, since recent spectroscopy (Sbarufatti et al. 2005b) has shown that the source is a broad-line QSO at $z=1.5$. Our sample is thus reduced to 106 objects.

2. For four objects 0426-380, 1519-273 (Sbarufatti et al. 2005a), 0754+100, and 1914-194 (Carangelo et al. 2003), new redshifts have been published in the last years, and for one 


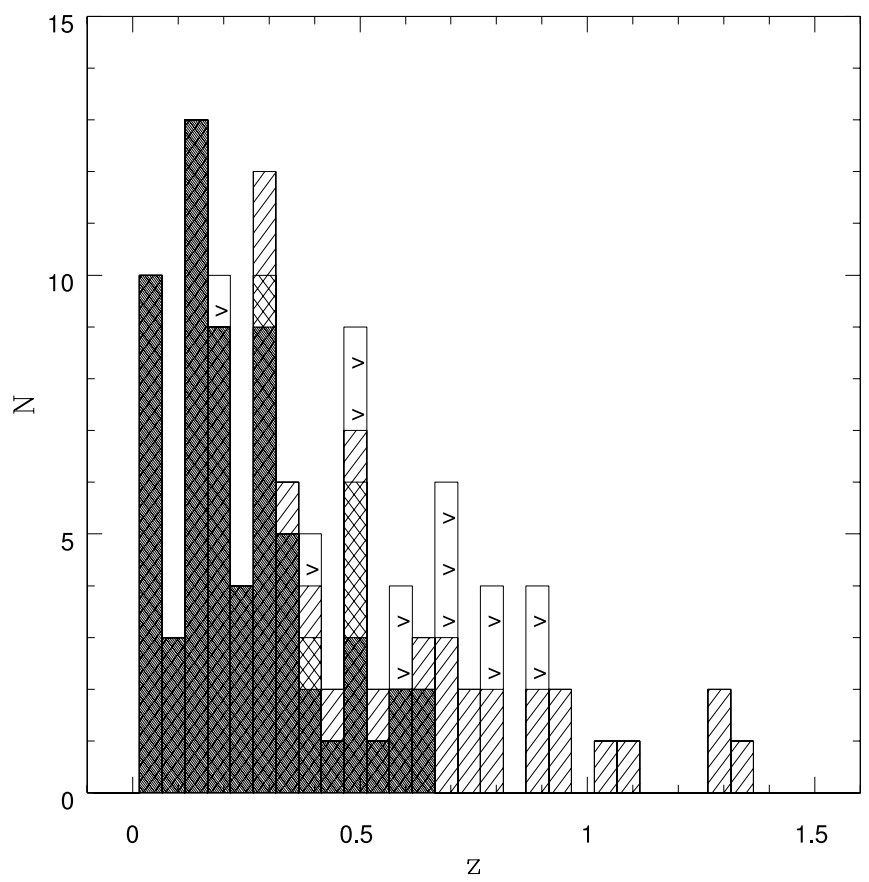

FIG. 1.-Redshift distribution of BL Lac objects in the HST snapshot imaging survey. Group A (host detected, redshift known): shaded area. Group B (host detected, redshift unknown): cross-hatched area. Group $\mathrm{C}$ (host undetected, redshift known): hatched area. Group D (host undetected, redshift unknown): open area with arrows. Lower limits are represented by arrows.

$(0158+001)$ the redshift was revised (Sloan Digital Sky Survey [SDSS]). ${ }^{2}$

3. The galactic extinction is now accounted for following Schlegel et al. (1998).

4. The $K$-correction for galaxy magnitudes is taken from Poggianti (1997).

5. The adopted cosmological parameters are $H_{0}=70 \mathrm{~km} \mathrm{~s}^{-1}$ $\mathrm{Mpc}^{-1}, \Omega_{\Lambda}=0.7$, and $\Omega_{m}=0.3$.

6. In order to compare the luminosity of the hosts at different redshifts (and epochs), we have also included a correction for taking into account the passive evolution of the galaxies following the Bressan et al. (1998) prescriptions. This correction, weighted on the redshift distribution, is $\sim 0.3 \mathrm{mag}$.

Table 1 reports the relevant parameters for the 106 objects. According to the spectra and imaging properties, the objects in the sample can be divided into four groups:

1. Objects for which the redshift is known from spectroscopy and the host galaxy is detected $(N=64)$. All the sources with $z \leq 0.2$ belong to this class (see Falomo et al. 2000).

2. Objects for which the host galaxy has been detected but with unknown redshift $(N=5)$.

3. Objects of known redshift but the host galaxy has not been detected $(N=24)$.

4. Objects of unknown redshift and that have not been resolved by HST optical imaging $(N=13)$.

The redshift distribution of these groups is given in Figure 1. As expected, the objects in group A are clustered at low redshift $(z<0.5)$, while those that are unresolved tend to be at $z>0.5$.

2 See SDSS Data Release 4, http://cas.sdss.org/astro/en/tools/getimg/spectra .asp, plate 403/51871, fiber 631, and Richards et al. (2002) for a description of the quasar survey.

\section{RESULTS}

\subsection{The Hubble Diagram of the Host Galaxies of BL Lac Objects}

The absolute magnitude of each host galaxy, modified for the effects of $K$ - and evolution correction (Poggianti 1997; Bressan et al. 1998), is reported in Table 1. The distribution of the absolute magnitude $\left(M_{R}\right)$ of the host galaxies for objects in group A is reported in Figure 2. This distribution is rather narrow and can be well approximated by a Gaussian with mean value $\left\langle M_{R}\right\rangle=$ -22.8 and standard deviation $\sigma_{M}=0.5$. Note that U00 reported $\left\langle M_{R}\right\rangle=-23.7 \pm 0.6$. The main difference is due to the difference in cosmological parameters (U00 used $H_{0}=50 \mathrm{~km} \mathrm{~s}^{-1}$ $\mathrm{Mpc}^{-1}, \Omega=0$ ) and the addition of the correction for the passive evolution of the galaxies. These variations also produce a smaller dispersion of the distribution $(0.5$ with respect to 0.6 given in U00).

In order to test how the host galaxy luminosity of BL Lac objects can be used as a standard candle, we have constructed a Hubble diagram. The relation between the apparent magnitude $m_{R}$ of the host galaxy and the redshift $z$ is given by

$$
m_{R}=M_{R}-K(z)+E(z)-5+5 \log d(z),
$$

where $M_{R}$ is the host absolute magnitude, $K(z)$ is the $K$-correction, $E(z)$ is the evolution correction, and $d(z)$ is the luminosity distance. With the assumptions described above for the cosmology and the $K$-and passive evolution corrections, the only remaining

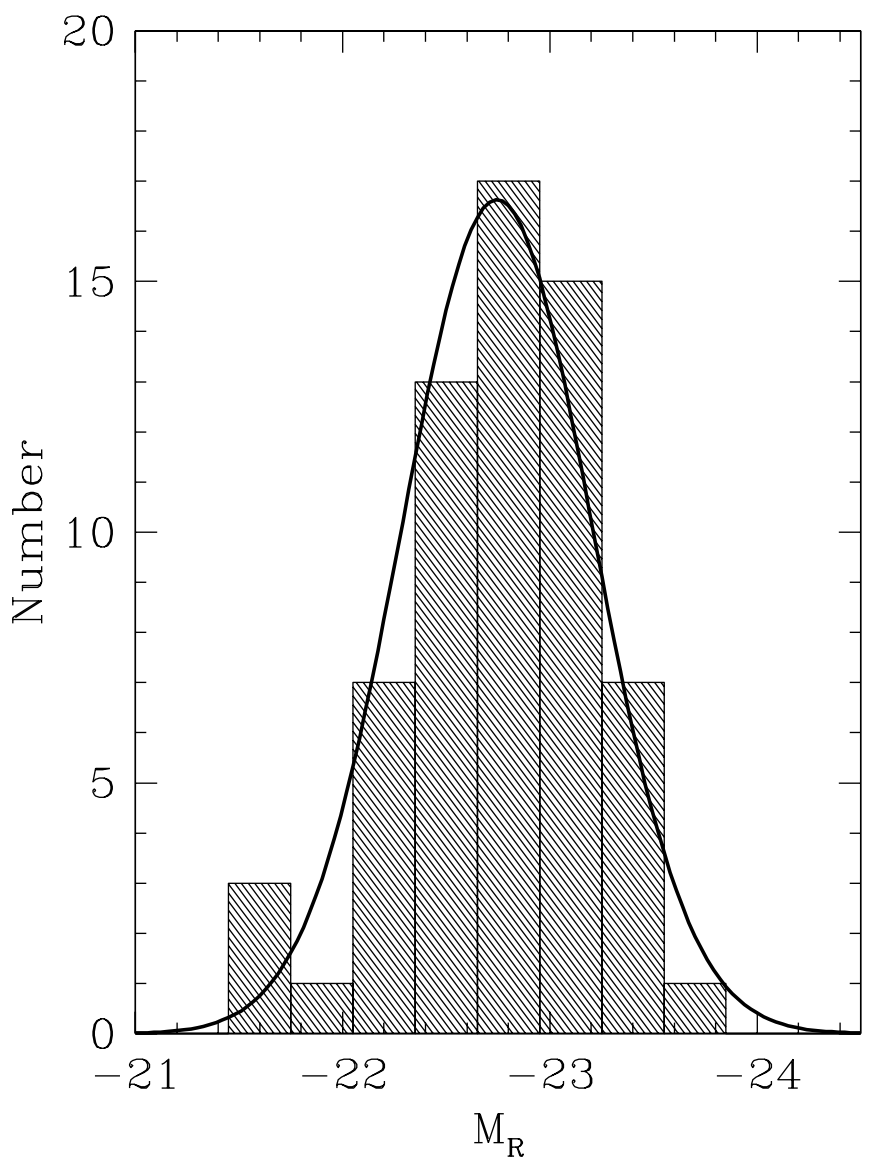

FIG. 2.-Distribution of the host galaxy absolute magnitude $M_{R}$ for objects of group A. The solid line represents a Gaussian fit to the distribution (mean $\left.M_{R}=-22.8, \sigma=0.5\right)$. 


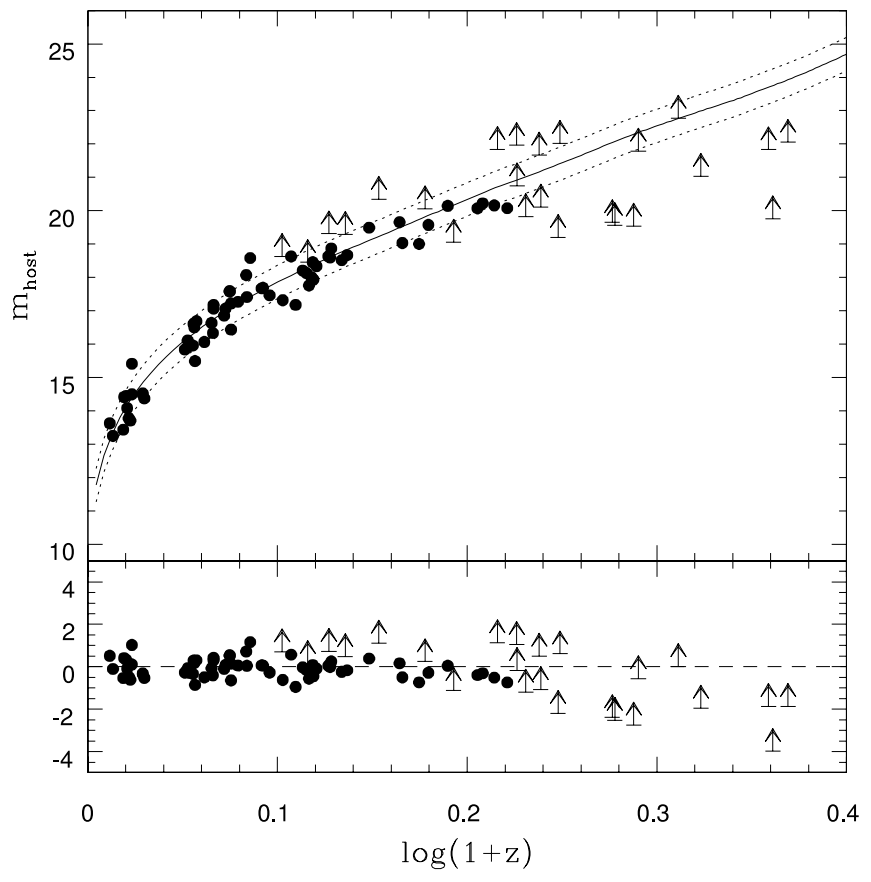

Fig. 3.-Top: Hubble diagram for host galaxies of BL Lac objects. Apparent magnitudes $(R$ filter) are corrected for the galactic extinction. Group A objects are represented by filled circles. Group $\mathrm{C}$ objects are shown as upper limits. The solid line corresponds to a fit with a galaxy of $M_{R}=M_{R}^{\text {fit }}=-22.9$. Dotted curves correspond to a host galaxy 0.5 mag brighter (bottom curve) or fainter (top curve). Bottom: Deviations of the data from the fit.

free parameter is the host magnitude $M_{R}$. The best fit of the observed data yields $M_{R}^{\text {fit }}=-22.9$. The Hubble diagram ( $m_{R}$ vs. $\left.z\right)$ for the BL Lac hosts together with the best fit is shown in Figure 3. It is notable that $\sim 70 \%$ of the points representing the resolved BL Lac objects are encompassed within $M_{R}^{\text {fit }} \pm 0.5$ mag (i.e., $-23.4<M_{R}<-22.4$ ). This Hubble diagram can be used to obtain a photometric redshift of the objects from the measurement of the host galaxy apparent magnitude, or a lower limit on $z$ only if a lower limit on the magnitude is available. In the redshift range considered here $(z \lesssim 0.7)$, the Hubble diagram can be well represented by the following expression:

$$
\log (1+z)=\left(0.293 m_{R}^{2}-7.19 m_{R}+45.1\right) \times 10^{-2},
$$

which approximates the Hubble diagram with a precision better than $1 \%$.

To evaluate the capability of this method we compare in Figure 4 the redshifts given by this photometric technique with the redshifts derived from the spectra in all cases in which they are available. The comparison shows that, apart from very few exceptions, the $z$ estimated by the host galaxy luminosity is in very good agreement with the one obtained spectroscopically. The average difference of redshift between the two methods is

$$
\langle\Delta z\rangle=0.01 \pm 0.05(\mathrm{rms}) .
$$

The main conclusion of this analysis is therefore that, at least in the explored redshift range $(z<0.7)$, the measurement of the apparent magnitude of the host galaxy of a BL Lac source allows one to estimate its redshift with an average accuracy of 0.05 . Because the U00 sample is unbiased as far as host galaxies are concerned, this technique can be of use any time that the $R$ apparent magnitude of a BL Lac host galaxy is measured, or a lower limit is obtained. In particular, equation (2) gives a

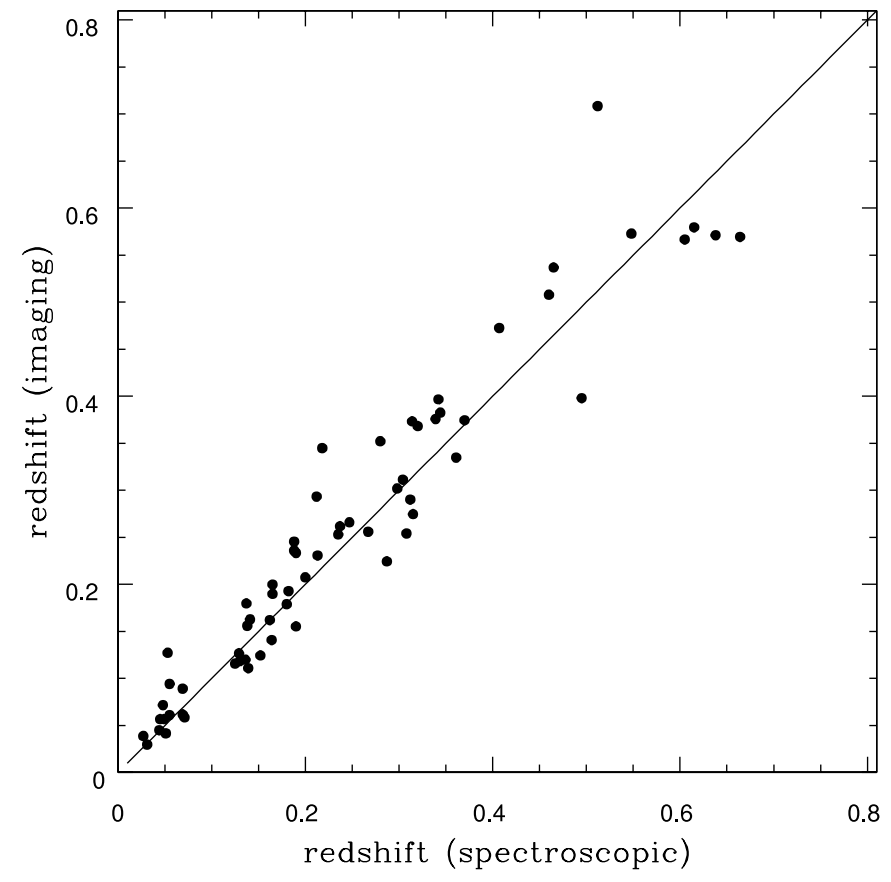

FIG. 4.-Comparison of imaging redshift obtained using the Hubble diagram fit and the spectroscopic redshift for the objects in group A. The solid line represents the one-to-one relation.

straightforward method for estimating the redshift in such cases. In the following sections we use this technique to derive the redshift or a lower limit for the objects in groups B, C, and D.

\subsection{Imaging Redshifts}

\subsubsection{Group B (Host Galaxy Detected, Unknown Spectroscopic Redshift)}

Using the fit derived above for the Hubble diagram, we can get a photometric estimate of the redshift for the five objects with hosts detected but no spectroscopic $z$ available (see Table 1; group B). The redshift of these objects ranges from $z=0.26$ to 0.54. Combining the uncertainty derived from equation (3) with the one corresponding to the host galaxy apparent magnitude (which is of the order of $0.1 \mathrm{mag}$ ), the error on the imaging redshifts turns out to be $\lesssim 0.1$. From the estimated $z$, one can then obtain the nucleus luminosity. For the nucleus we adopted a $K$-correction following Wisotzki (2000), under the hypothesis that its optical spectrum is described by a power law $\left(F_{\lambda} \propto \lambda^{-\alpha}\right.$ with $\alpha=0.7$; see Falomo et al. 1994). Results are reported in Table 1 .

\subsubsection{Group C (Redshift Known, Host Undetected)}

From the HST images of these sources we have a lower limit on the magnitude of the host reported by U00 (see Table 1), and we know the redshift from the spectra. From these values we can obtain a lower limit on the absolute magnitude of the host galaxy. These limits are in most cases consistent (see Fig. 3) with the presence of a host galaxy less luminous than $M_{R}=-23.4$ (i.e., $\left.M_{R}^{\text {fit }}-0.5\right)$. In no case is the derived limit for the host luminosity lower than $M_{R}=-21.9$ (i.e., $M_{R}^{\text {fit }}+1.0$ ). Therefore, the fact that the host galaxy has not been detected in these HST images is consistent with the capability of the observation and with a distribution of the host absolute magnitude as given in Figure 2. The nondetection of the host in most cases is likely due to a high nuclear-to-host galaxy ratio (N/H; see $\S 4$ and Fig. 6). 


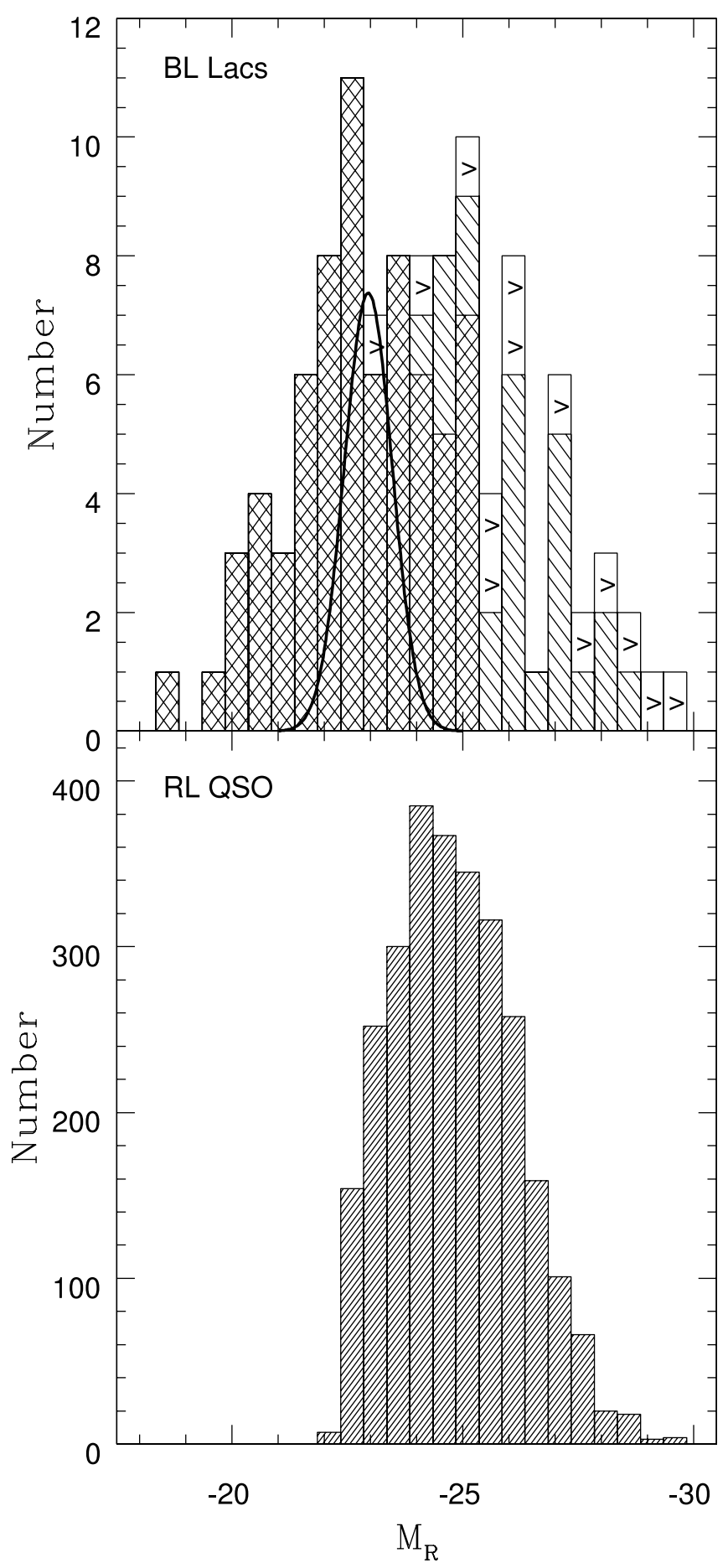

FIG. 5.-Top: Distribution of nucleus absolute magnitude of BL Lac objects in the $R$ filter. Groups A and B: cross-hatched area. Group C: hatched area. Group D: open area with arrows. The solid line represents the Gaussian fit to the host galaxy absolute magnitude distribution for group A, scaled by a factor of 0.5 . Upper limits are represented by arrows. Bottom: Distribution of absolute $R$ magnitudes for radio-loud quasars (RL QSOs) in the Véron-Cetty \& Véron (2003) catalog with $z<1.4$.

\subsubsection{Group D (No Host, No Redshift: Extreme BL Lac Objects)}

The remaining group of objects is that formed by the unresolved sources that exhibit a featureless spectrum. The redshifts of these objects are thus far still unknown. The only information that can be derived from the images is therefore the brightness of the nucleus and an upper limit to the brightness of the surrounding nebulosity. Assuming also that these objects are hosted in a galaxy with $M_{R}=M_{R}^{\text {fit }}=-22.9$ and from the lower limit of the magnitude of the surrounding nebulosity, one can derive a lower limit to the redshift using the Hubble diagram (see Fig. 3 and Table 1). This lower limit to the redshift can then be used to derive a lower limit to the luminosity of the nucleus. The distribution of the absolute magnitude of the nuclei for all objects in the four groups is shown in Figure 5. It turns out that most of the sources in group D are more luminous than $M_{R}=-25$ and fill the bright tail of the luminosity distribution. It is also worth noting that of the 13 objects in this group, 10 are HBL and only three are LBL, while in the whole sample there are $71 \mathrm{HBL}$ and 35 LBL.

\section{DISCUSSION: NUCLEAR LUMINOSITIES AND BEAMING}

We have shown that the detection of the host galaxy allows a photometric determination of the redshift for the five objects in group B. Since these objects are at $0.2<z<0.5$, they are convenient targets for redshift measurement through spectroscopy. Direct estimates of the absolute nuclear magnitude are available for those objects with known spectroscopic redshift (group A and $C$ ). In addition, using the imaging redshifts, we can add five more targets (group B objects) and 13 upper limits for the objects in group D. The redshift distribution of the whole sample is given in Figure 1.

The absolute nuclear magnitudes are summarized in Table 1. The mean and median absolute magnitude for objects in groups A-C are $M_{R}=-23.7$ and -23.6 , respectively, while adding objects in group D the median is $M_{R}=-24.1$. As already noted by U00, the distribution of the absolute magnitude of the host galaxies is much narrower than that of their nuclei (Fig. 5).

Since the objects in each individual sample used to produce the HST snapshot survey are selected on the basis of their radio and X-ray fluxes, it is expected that the entire data set of objects suffers from the typical bias of flux-limited surveys. Nevertheless, some interesting comments should be made. Our analysis of the redshift and the nuclear luminosity of the objects clearly shows that the extreme BL Lac objects (group D, those objects with featureless spectra and unresolved) are among the most luminous sources in the sample. Indeed, 10 of the 13 objects are brighter than the brightest object in group A (see Fig. 5, top). If one compares the luminosity distribution with that of $z<1.4$ radio-loud quasars (using homogeneous corrections for the magnitudes) used for the BL Lac objects, it is apparent that the bright tail of the BL Lac distribution is consistent with that of normal radio-loud quasars (see Fig. 5, bottom).

Below the absolute magnitude $M_{R}=-25$ there are basically two types of objects, groups C and D. Unless group D is really significantly more luminous than the average of objects in group $\mathrm{C}$ (objects with emission lines), the different spectral properties could be related to different amounts of beaming. If one assumes that the two types of objects have a similar broad-line region, the strength of the line should be related to the intrinsic ionizing flux. If blazars behave as normal quasars, the line emission should depend on the unbeamed continuum (e.g., Pian et al. 2005 and references therein). Under this hypothesis we therefore argue that in extreme BL Lac objects the mechanism for emission-line formation is less efficient because the intrinsic continuum source is smaller with respect to group $\mathrm{C}$ objects, but more strongly beamed.

This suggestion is made stronger by the consideration of the distribution of the objects in the plane $m_{R}$ versus $z$ (see Fig. 6). The curves represent the loci of a constant $\mathrm{N} / \mathrm{H}$, defined as the ratio of monochromatic $R$-band luminosities in the object rest 


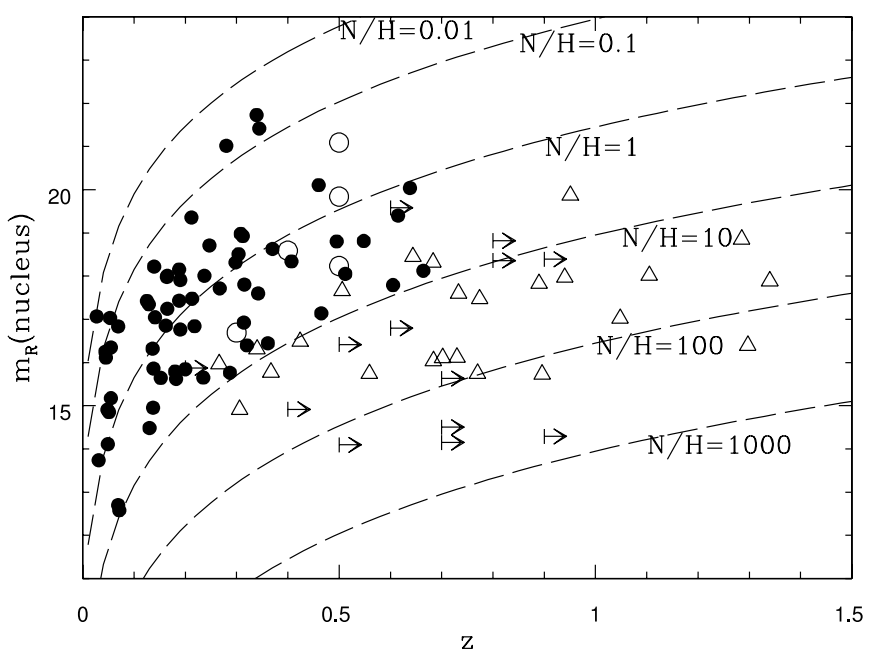

FIG. 6.-Distribution of nucleus apparent magnitude (corrected for galactic extinction) vs. redshift. Dashed lines show the loci of constant $\mathrm{N} / \mathrm{H}$, assuming a host galaxy with $M_{R}=M_{R}^{\text {fit }}=-22.9$. Group A objects are represented by filled circles. Group B objects are represented by open circles (redshift estimated using Hubble diagram. Group C objects are represented by triangles. Group D objects are represented by arrows (lower limits on redshift determined using Hubble diagram).

frame. All the resolved sources are in the region with $\mathrm{N} / \mathrm{H}$ less than 10, while the large majority of objects in groups $\mathrm{C}$ and $\mathrm{D}$ are in the region between $\mathrm{N} / \mathrm{H}=10$ and 1000. Again, in this context group $\mathrm{D}$ objects are clearly the most extreme, implying high $\mathrm{N} / \mathrm{H}$ (sometimes in excess of $\mathrm{N} / \mathrm{H}=100$ ). It is also notable that group D objects are mostly of the HBL type, while the majority of group C objects are of the LBL type. The inference is therefore that in the optical band HBLs are more beamed than LBLs.

Independent information on the amount of beaming can be derived from the broadband spectral energy distribution, assuming that the emitted continuum is basically the superposition of a synchrotron and an inverse Compton component (see, e.g., Ghisellini et al. 1998). Thus far, these studies were limited to objects of known redshifts, which are irrelevant to our proposal that extreme BL Lac objects are extremely beamed objects, but some extension to this class of sources may soon become available.

\section{CONCLUSIONS}

We have reanalyzed the host galaxies and nuclear properties of the BL Lac objects observed by HST snapshot imaging survey. The magnitudes of the objects in the data set have been revised according to the treatment of the galactic extinction and the evolution and $K$-corrections. The concordant cosmological parameters have been used.

The main results and conclusions from this study are as follows:

1. The host galaxy absolute magnitude distribution is sufficiently narrow (Gaussian distribution centered around $M_{R}=$ -22.9) that the host galaxy can be used as a standard candle to derive the photometric redshift of the objects. Therefore, a determination of $z$ (or a lower limit) can simply be derived from the measurement (or from the lower limit) of the host galaxy apparent magnitude.

2. The determination of the redshift and the lower limits allows us to investigate the nuclear luminosity distributions for various type of objects. This suggests that the objects in the sample that are unresolved and characterized by a featureless spectrum are the most luminous and/or beamed nuclei of the class. These extreme BL Lac objects could have a nucleus-to-host galaxy ratio of 100 to 1000 .

This work was supported in part by the Italian Space Agency $(\mathrm{I} / \mathrm{R} / 056 / 02)$ and the Italian Ministery of Education COFIN 2004023189
Abraham, R. G., Crawford, C. S., \& McHardy, I. M. 1991, MNRAS, 252, 482 Blandford, R. D., \& Rees, M. J. 1978, in Pittsburgh Conference on BL Lac Objects, ed. A. M. Wolfe (Pittsburg: Univ. Pittsburgh Press), 328

Bressan, A., Granato, G. L., \& Silva, L. 1998, A\&A, 332, 135

Carangelo, N., Falomo, R., Kotilainen, J., Treves, A., \& Ulrich, M.-H. 2003, A\&A, 412, 651

Falomo, R. 1996, MNRAS, 283, 241

Falomo, R., \& Kotilainen, J. K. 1999, A\&A, 352, 85

Falomo, R., Scarpa, R., \& Bersanelli, M. 1994, ApJS, 93, 125

Falomo, R., Scarpa, R., Treves, A., \& Urry, C. M. 2000, ApJ, 542, 731

Falomo, R., Urry, C. M., Pesce, J. E., Scarpa, R., Giavalisco, M., \& Treves, A. 1997, ApJ, 476, 113

Ghisellini, G., Celotti, A., Fossati, G., Maraschi, L., \& Comastri, A. 1998, MNRAS, 301, 451

Heidt, J., Nilsson, K., Sillanpää, A., Takalo, L. O., \& Pursimo, T. 1999, A\&A, 341,683

Heidt, J., Tröller, M., Nilsson, K., Jäger, K., Takalo, L., Rekola, R., \& Sillanpää, A. 2004, A\&A, 418, 813

Nilsson, K., Pursimo, T., Heidt, J., Takalo, L. O., Sillanpää, A., \& Brinkmann, W. 2003, A\&A, 400, 95

O’Dowd, M., \& Urry, C. M. 2005, ApJ, 627, 97

Padovani, P., \& Giommi, P. 1995, ApJ, 444, 567

\section{EFERENCES}

Pian, E., Falomo, R., \& Treves, A. 2005, MNRAS, 361, 919

Poggianti, B. M. 1997, A\&AS, 122, 399

Richards, G. T., et al. 2002, AJ, 123, 2945

Romanishin, W. 1987, ApJ, 320, 586

Sbarufatti, B., Treves, A., Falomo, R., Heidt, J., Kotilainen, J., \& Scarpa, R. 2005a, AJ, 129, 599 2005b, AJ, submitted

Scarpa, R., Urry, C. M., Falomo, R., Pesce, J. E., \& Treves, A. 2000a, ApJ, 532,740

Scarpa, R., Urry, C. M., Padovani, P., Calzetti, D., \& O’Dowd, M. 2000b, ApJ, 544,258

Schlegel, D. J., Finkbeiner, D. P., \& Davis, M. 1998, ApJ, 500, 525

Sowards-Emmerd, D., Romani, R. W., Michelson, P. F., Healey, S. E., \& Nolan, P. L. 2005, ApJ, 626, 95

Véron-Cetty, M.-P., \& Véron, P. 2003, A\&A, 412, 399

Urry, C. M., Falomo, R., Scarpa, R., Pesce, J. E., Treves, A., \& Giavalisco, M. 1999, ApJ, 512, 88

Urry, C. M., Scarpa, R., O’Dowd, M., Falomo, R., Pesce, J. E., \& Treves, A. 2000, ApJ, 532, 816 (U00)

Wisotzki, L. 2000, A\&A, 353, 861

Wurtz, R., Stocke, J. T., \& Yee, H. K. C. 1996, ApJS, 103, 109 